\title{
An Unusual Presentation of Cryptococcal Infection in a Post Renal Transplant Patient: A Case Report
}

\author{
Wickrama Arachchi Appuhamilage Gayani Nadeesha Nishanthi ${ }^{1, *}$, and Ahmed Shiyam² \\ ${ }^{1}$ Senior Registrar in Nephrology, Dialysis and transplant centre, Sri Jayawardenapura General Hospital, Sri Lanka \\ ${ }^{2} \mathrm{MD}$ Trainee, Postgraduate Institute of Medicine, University of Colombo, Ministry of Health, Sri Lanka
}

*Corresponding authors: Wickrama Arachchi Appuhamilage Gayani Nadeesha Nishanthi, Senior Registrar in Nephrology, Dialysis and transplant centre, Sri Jayawardenapura General Hospital, Sri Lanka, E-mail: littlenadee@yahoo.com

Received: 19 Apr, 2021 | Accepted: 27 May, 2021 | Published: 03 Jun, 2021

Citation: Nishanthi WAAGN, Shiyam A (2021) An Unusual Presentation of Cryptococcal Infection in a Post Renal Transplant Patient: A Case Report. J Clin Case Stu 6(2): dx.doi.org/10.16966/2471-4925.221

Copyright: (c) 2021 Nishanthi WAAGN, et al. This is an open-access article distributed under the terms of the Creative Commons Attribution License, which permits unrestricted use, distribution, and reproduction in any medium, provided the original author and source are credited.

\section{Abstract}

Background: Cryptococcus neoformans is opportunistic yeast commonly found in soil contaminated by bird feces throughout the world. Cryptococcosis is a severe fungal infection in a with a high mortality rate among solid organ transplant recipients. The infection usually involves the lungs, central nervous system, skin, bones, and the urinary tract. Cryptococcosis continues to cause significant mortality and morbidity in immunocompromised patients.

Case presentation: A 40-year-old man, who underwent renal transplantation 6 years before, presented with early morning severe headache, nausea, slurring of speech and unsteadiness of walk for 2 weeks' duration. On examination he had some cushinoid features and dilated superficial veins in the chest and neck.

Conclusions: Patient's symptoms fully recovered within first 2 weeks of the treatment and his repeat lumbar puncture after 2 weeks' treatment was completely negative. The main learning point of this case presentation is good history and proper clinical examination is very important in an immunocompromised patient to make the correct diagnosis.

Keywords: Cryptococcus neoformans; Renal transplantation; Case report

\section{Introduction}

Cryptococcus neoformans is opportunistic yeast commonly found in soil contaminated by bird feces throughout the world. Cryptococcosis continues to cause significant mortality and morbidity in immunocompromised patients. It is third in the frequency after Aspergillus and Candida $[1,2]$ cryptococcal infection usually occurs 1 year after transplantation as Fluconazole prophylaxis usually not given later. The overall incidence of crytococcosis in SOT recipients is $2.8 \%$ with mortality rate around $40 \%$. In this case report we discuss the rare clinical presentation of cryptococcal infection in a post renal transplant patient and his diagnosis and treatment.

\section{Case Presentation}

A 40-year-old man underwent renal transplantation on 2010 January, primary disease was IgA nephropathy, and mother was the donor with HLA mismatch 1:1:1 and panel reactive antibodies were negative. Immunosuppression protocol involved induction with IV Basiliximab, IV Methylprednisolone pulses for 3 days followed up with triple immunosuppressive therapy include prednisolone, MMF and Cyclosporin. He had developed NODAT 1 year following renal transplantation. He presented with early morning severe headache with nausea, slurring of speech and unsteadiness of walk for 2 weeks' duration. $\mathrm{He}$ also had recurrent presyncopal episodes and initially managed as diabetic autonomic neuropathy. He denied any history of fever. He had been taking oral prednisolone $5 \mathrm{mg}$ mane, Ciclosporin $75 \mathrm{mg}$ bd, Mycophenolic Mofetil Acid 500mg bd, and oral Gliclazide 40mg bd and Amlpdipine 5mg bd.

On examination he had some cushinoid features and dilated superficial veins in the chest and neck. His pembertan sign was positive. Fundi revealed Grade II hypertensive retinopathy Laboratory investigations revealed serum creatinine $2.9 \mathrm{mg} / \mathrm{dl}$ with baseline creatinine $1.6 \mathrm{mg} / \mathrm{dl}$, ESR-100mm/1st hour, WBC-8600, Hb-9.5g/dl, plt-229000, Chest X-ray revealed coin lesion in the right middle lobe and HRCT revealed right lung mass with bilateral lung nodules and mediastinal lymphadenopathy (Figure 1).

Blood culture and ABST-yeast isolated with Indian ink stain weekly positive, Serum for Cryptococcal antigen positive, Lym- $80 \%$, As CSF full report showed lymphocytic predominant high protein level it is suggestive of a fungal infection. His CSF for indian ink stain and cryptococcal antigen was positive (Figure 2).

First we have stopped MMF, reduced Cyclosporin 50mg bd and continued prednisolone $5 \mathrm{mg}$. We have treated him with intravenous liposomal Amphotericin B $(5 \mathrm{mg} / \mathrm{kg} /$ day dissolved in 5\% Dextrose for first 2 weeks followed by oral Fluconazole $800 \mathrm{mg} /$ day for 8 weeks followed by $400 \mathrm{mg} /$ day for 6 months. He required $4.75 \mathrm{~g}$ cumulative 


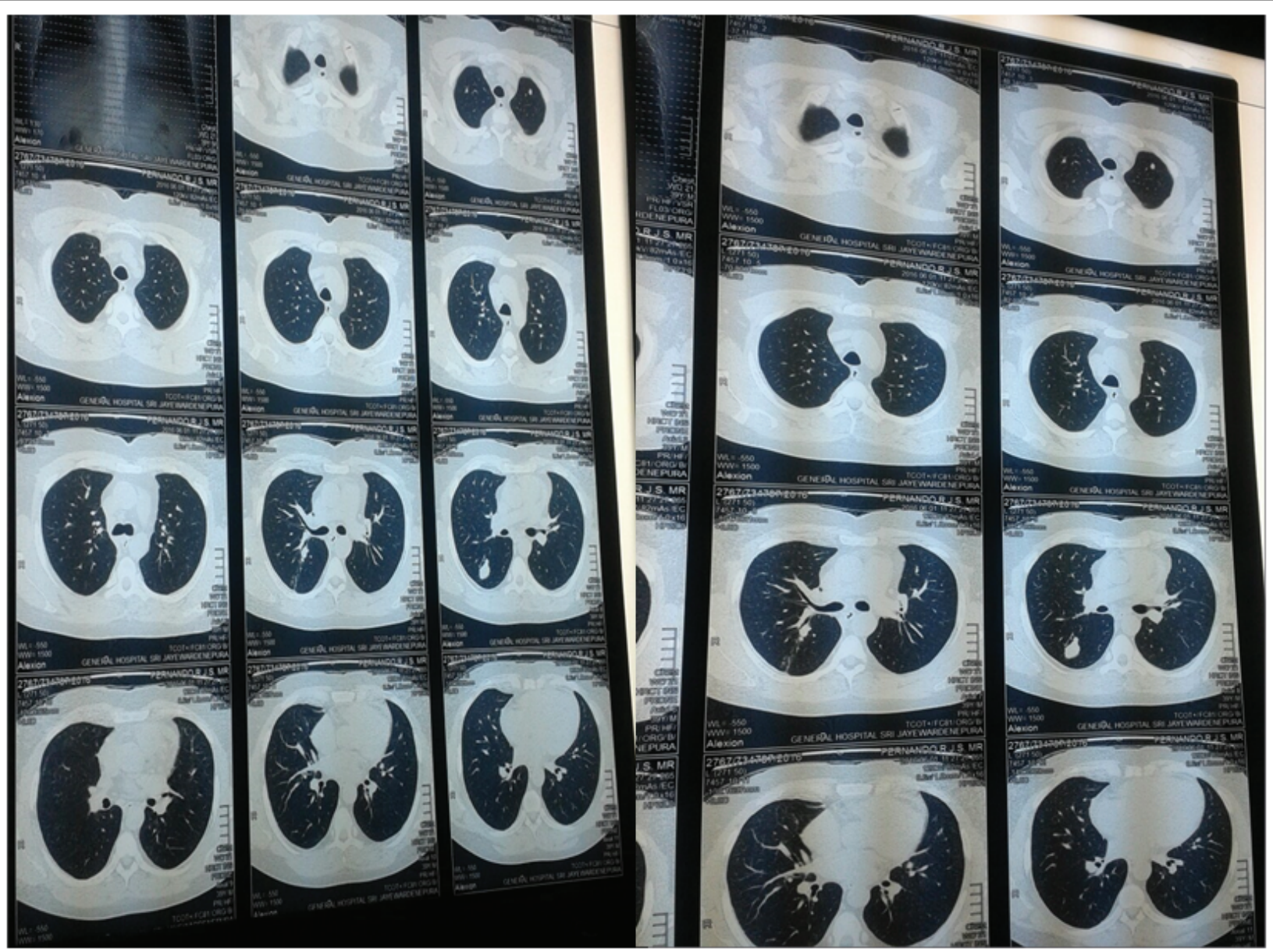

Figure 1: Chest X-ray and HRCT.

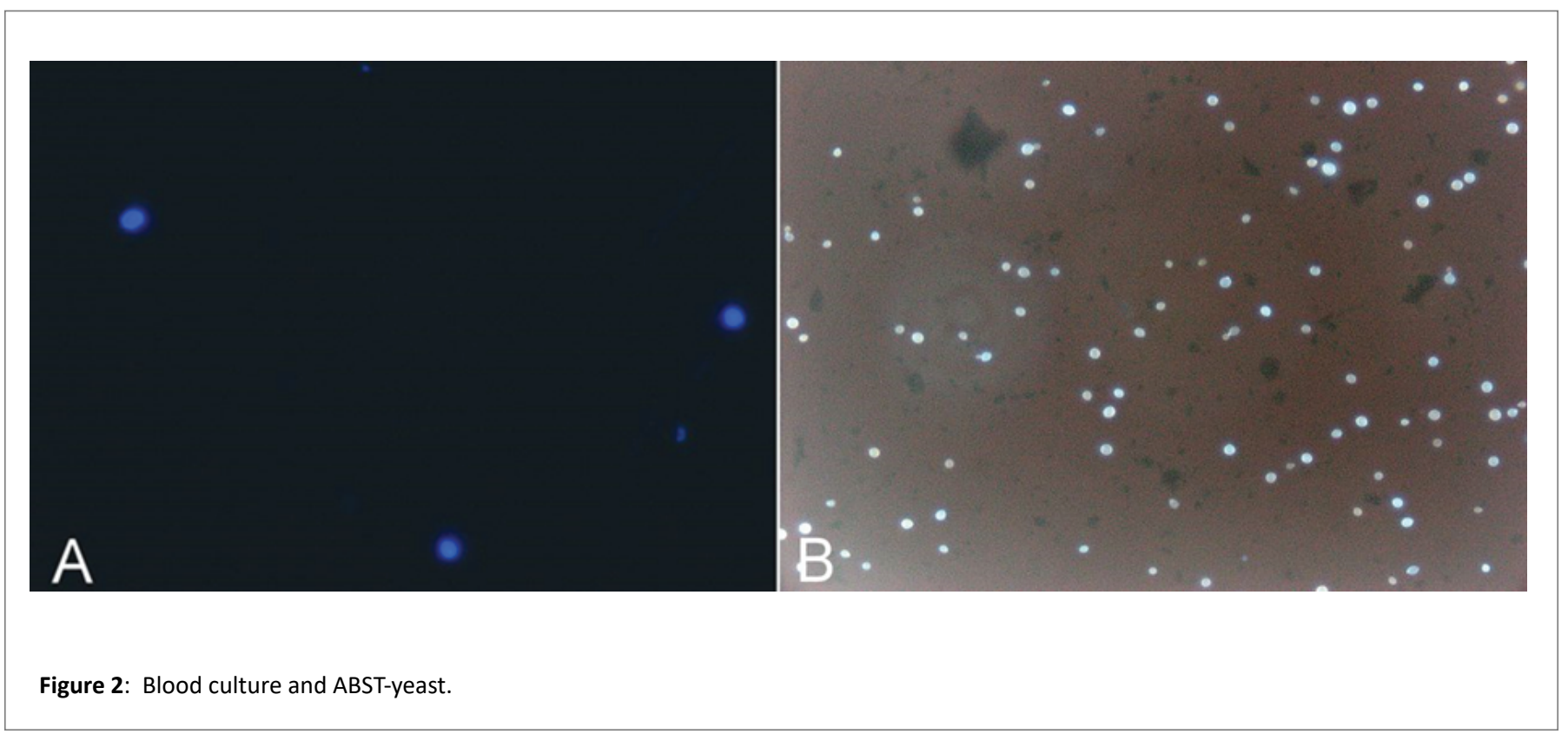

Citation: Nishanthi WAAGN, Shiyam A (2021) An Unusual Presentation of Cryptococcal Infection in a Post Renal Transplant Patient: A Case Report. J Clin Case Stu 6(2): dx.doi.org/10.16966/2471-4925.221 
dose of liposomal amphotericin B. We have corrected his hypokalemia during treatment. We couldn't treat with intravenous Flucytosine initially as it was unavailable in Srilanka. Patient's symptoms fully recovered within first 2 weeks of the treatment and his repeat lumbar puncture after 2 weeks' treatment was completely negative. Then he was treated with oral Flucanazole $800 \mathrm{mg} /$ day for 8 weeks followed by $400 \mathrm{mg} /$ day for 6 months. He was continued on oral prednisolone, cyclosporine and MMF was changed to Azathioprine with oral hypoglycemic drugs and antihypertensives. His serum creatinine was stable around $1.6 \mathrm{mg} / \mathrm{dl}$ which was his baseline. Patient's clinical condition became much better and is kept in regular transplant clinic follow up.

\section{Discussion and Conclusion}

Cryptococcosis remains a significant opportunistic infection in solid organ transplant recipients. It is encapsulated, basidiomycetous yeast that is present in the environment that has been isolated from pigeon droppings [3]. Cryptococcal disease is the third most common fungal infection after Aspergillus and Candidiasis in solid organ transplant recipients [4]. Majority of cryptococcal infection was considered due to reactivation of latent infection in immunocompromised state [1]. A total of $53 \%-72 \%$ of cases of solid organ transplant recipients are disseminated or involve the CNS. It has preferred to invade CNS and meningitis is the most common first incidence of clinical presentation. It usually presents 16-21 months after renal transplantation [2]. Overall $61 \%$ of the SOT recipients had disseminated disease, $54 \%$ had pulmonary disease, and $8.1 \%$ had skin, soft tissue or osteoarticular cryptococcosis. The most sensitive and specific test for diagnosis of Cryptococcal infection was indian ink staining of the aspirate and it reveals encapsulated yeast cells [5]. Additional investigations include identification of organism by culture. The latex agglutination and enzyme immune assay for serum and CSF cryptococcal antigens is excellent rapid diagnostic test. In our case serum and CSF cryptococcal antigen became positive which benefits to initiate treatment sooner.

Calcineurin inhibitors can affect the extent of the disease and steroids are associated with increased risk of infection [6,7]. However, Basiliximab which we have used for induction at the transplant is not associated with increased risk of infection compared to other induction agents [8]. Induction treatment in patients with disseminated infection should include liposomal Amphotericin B with Flucytosine.

The main learning point of this case presentation is good history and proper clinical examination is very important in an immunocompromised patient to make the correct diagnosis. When a patient presented with presyncopal episodes, it should not be misdiagnosed as diabetic autonomic neuropathy without doing proper clinical examination.

\section{Declaration}

I declare that this is an original research and there is no duplication, and I didn't publish it in any other journals.

\section{Ethics Approval and Consent to Participate}

Ethical clearance was obtained from Sri Jayawardenapura General Hospital Ethical Committee.

\section{Consent}

Consent to publish I'm giving the consent to publish BMC Nephrology journal and the full text and images can be freely available on the internet.

\section{Availability of Data and Materials}

The datasets used and analysed during the current study are available from the corresponding author on reasonable request.

\section{Competing Interests}

There is no competing of interests.

\section{Funding}

Self-funded by first author.

\section{Acknowledgements}

The authors appreciate all people that patiently contributed to this study.

\section{Contributions}

NWAAGN was responsible for the conception, design of the article, data collection, and follow-ups. NWAAGN and AS were responsible for interpretation and writing of the paper. All authors read and approved the final manuscript.

\section{References}

1. Garcia-Hermoso D, Janbon G, Dromer F (1999) Epidemiological evidence for dormant Cryptococcus neoformans infection. J Clin Microbiol 37: 3204-3209.

2. Singh N, Alexander BD, Lortholary O, Dromer F, Gupta KL, et al. (2007) Cryptococcus neoformans in organ transplant recipients: impact of calcineurin-inhibitor agents on mortality. J Infect Dis 195: 756-764.

3. Soltani M, Bayat M, Hashemi SJ, Zia M, Pestechian N (2013) Isolation of Cryptococcus neoformans and other opportunistic fungi from pigeon droppings. J Res Med Sci 18: 56-60.

4. Pappas PG, Alexander BD, Andes DR, Hadley S, Kauffman CA, et al. (2010) Invasive fungal infections among organ transplant recipients: results of the Transplant-Associated Infection Surveillance Network (TRANSNET). Clin Infect Dis 50: 1101-1111.

5. Gupta RK, Khan ZU, Nampoory MRN, Mikhail MM, Johny KV (2004) Cutaneous cryptococcosis in a diabetic renal transplant recipient. J Med Microbiol 53: 445-449.

6. Singh N, Forrest G, AST Infectious Diseases Community of Practice (2009) Cryptococcosis in solid organ transplant recipients. Am J Transplant 9: S192-S198.

7. Dromer F, Mathoulin-Pélissier S, Fontanet A, Ronin O, Dupont B, et al. (2018) Epidemiology of HIV-associated cryptococcosis in France (1985-2001): comparison of the pre- and post-HAART eras. AIDS 18: 555-562.

8. Burdette SD, Sun HY, Infections Associated with Specific Immunosuppressive Agents in Transplant Recipients. 